\title{
Intracerebral Hemorrhage: Mechanisms and Therapies
}

\author{
Guohua Xi • Richard F. Keep
}

Received: 24 April 2012 / Accepted: 26 April 2012 /Published online: 11 May 2012

(C) Springer Science+Business Media, LLC 2012

We welcome you to this special issue of Translational Stroke Research on intracerebral hemorrhage (ICH). There are about two million cases of ICH in the world each year, accounting for about $10-15 \%$ of all strokes in the USA, Europe, and Australia and $20-30 \%$ of strokes in Asia. It has a very high mortality rate $(\sim 40 \%)$, and most survivors face long-term disability $[1,2]$. Despite this, ICH has historically received much less focus than ischemic stroke. However, in the last years, there has been a marked increase in interest. Thus, Medline reported 374, 442, 590, 945, and 1,614 articles on intracerebral hemorrhage in the years from 1986-1990, 1991-1995, 1996-2000, 2001-2005, and 2006-2010, respectively. This upswing in interest is reflected by this special issue which includes 19 papers by leading experts in the field including clinical and preclinical studies, reviews and new research, and three editorials.

Rincon and Mayer [3] provide an extensive clinical overview of current management of ICH as well as ongoing clinical trials. Those trials include several aimed at clot evacuation with or without the use of tissue plasminogen activator (tPA) to aid in clot lysis $[4,5]$. Tissue plasminogen activator is also being examined in the CLEAR IVH trial [6] for intraventricular hemorrhage (IVH). Ventricular extension worsens ICH-induced injury [7]. In this issue, Strahle et al. review evidence on the mechanisms of IVH-induced injury [8]. This is particularly important in relation to hemorrhage of the germinal matrix in premature neonates.

G. Xi $(\square) \cdot$ R. F. Keep $(\square)$

Department of Neurosurgery, University of Michigan,

Ann Arbor, MI, USA

e-mail: guohuaxi@umich.edu

e-mail: rkeep@umich.edu
Neonatal hemorrhage is also the subject of an editorial by Bouz [9].

In treating cerebral ischemia with tPA, patients developing ICH is a major concern. Similarly, treating ICH patients with hemostatic agents to prevent hematoma expansion may cause thromboembolic events [10]. In addition, as discussed by Fisher et al. in this issue, ischemic and hemorrhagic cerebrovascular disease frequently coexists in the same patient (mixed cerebrovascular disease) raising a conundrum of how to treat such patients. Fisher et al. discuss potential ways to solve that problem [11].

Questions have also been raised over whether tPA may have some adverse consequences in the brain including causing delayed edema formation. In this issue, Keric et al. present data in a porcine ICH model that plasminogen activator inhibitor-1 can reduce that edema formation [12].

Polymorphisms in the APOE gene are associated with the risk of developing ICH and prognosis after ICH $[13,14]$. In this issue, Lei et al. [15] report finding an interaction between APOE polymorphism and gender in determining ICH-induced injury. TOMM40 is a gene in close proximity to APOE. Valant et al. have examined the role of TOMM40 in cerebral amyloid angiopathy [16]. They report that single nucleotide polymorphisms in TOMM40 are associated with vascular amyloid deposition but not ICH.

The mechanisms that appear to underlie ICH injury differ substantially from those of cerebral ischemia [17]. Indeed, there is evidence that ischemia plays little role in most ICH [17], and it is dangerous to infer a potential therapeutic approach from one type of stroke to another. A case in point is hyperglycemia where there has been considerable interest in regulating glucose levels in ischemic patients (e.g., the GASP and current SHINE clinical trials; [18]) but, as indicated by the study of Feng et al., the evidence for a role of 
hyperglycemia in exacerbating ICH-induced brain injury is much less clear [19]. This deserves further investigation.

Perihematomal edema is a hallmark of ICH. In this issue, Bodmer et al. [20] review the molecular mechanisms underlying edema formation and potential therapeutic targets to reduce formation. ICH causes blood-brain barrier disruption and vasogenic edema formation. Liu and Sharp also review their work on the role of the Src family of kinases in bloodbrain barrier disruption (and repair) as well as in neuronal cell death [21]. Lok et al. describe experiments indicating that neuregulin may be a novel mechanism to reduce bloodbrain barrier disruption after brain injury [22].

Much work is ongoing in relation to the role of inflammation in ICH-induced neural and vascular injury. In this issue, Yao and Tsirka review the role of chemokines and particularly monocyte chemoattractant protein-1 (CCL2) in the influx of leukocytes into the brain after ICH and hemorrhagic brain injury [23]. Hammond et al. report their results on the types of inflammatory cells that infiltrate the brain after ICH and the changes in inflammatory mediators that may drive that infiltration [24]. Similarly, Lively and Schlichter examine the evolution of the inflammatory response after ICH by measuring inflammatory mediators levels [25]. They have found differences between young and old rats which may be important since ICH is mostly a disease of the elderly. Loftspring et al. examine the potential role of neutrophils in ICH-induced injury, but found no effect of neutrophil depletion [26]. Simard et al. used a different approach to manipulate inflammation in subarachnoid hemorrhage, systemic delivery of heparin [27]. They found significantly reduced neuroinflammation, demyelination, and apoptosis.

While it is well known that there is a profound astrocyte reaction after $\mathrm{ICH}$, less attention has been paid to this cell type as a potential therapeutic target. Here, SukumariRamesh et al. discuss the potential beneficial and detrimental effects of ICH-induced astrogliosis [28].

Advances in our understanding of ICH will be assisted by new methodologies. Two examples are presented in this edition. Hasan et al. present data using ferumoxytylenhanced MRI to image inflammation in human brain arteriovenous malformations (AVMs) [29]. This promises to be a new diagnostic tool for potential AVM rupture and a method of studying the natural course of AVM development. Xie et al. examine the vascular response after mouse $\mathrm{ICH}$ using micro $\mathrm{CT}$ after vascular perfusion with microfil [30]. This technique allows $3 \mathrm{D}$ vascular reconstructions in order to examine vasodilation and neovascularization in relation to the hematoma.

The potential of mixed cerebrovascular disease, the desire to treat patients as early as possible after ictus when it may not be certain whether a stroke is hemorrhagic or ischemic, and the possibility that some potential therapies may have beneficial and detrimental effects in different forms of stroke mean that it is important to test strategies in both ischemic and hemorrhagic stroke models. The effects of conditioning stimuli (induced tolerance) on ischemic brain damage are well known and are proceeding to clinical trial. The effects of ischemic preconditioning (IPC) on ICH-induced injury have not been studied. In this issue, $\mathrm{He}$ et al. show that IPC can reduce ICH-induced injury, although there were some effects on hemostasis that require further investigation [31].

As yet, there are still no therapies for ICH. However, as emphasized by the editorial by Morgenstern in this issue, there are now multiple clinical trials aimed at rectifying that situation, and this issue as a whole shows some of the breadth of work being undertaken to try and improve ICH treatment [32]. We would like to thank the contributors for the effort and time they put into this issue.

\section{References}

1. Adeoye $\mathrm{O}$, Broderick JP. Advances in the management of intracerebral hemorrhage. Nat Rev Neurosci. 2010;6(11):593-601.

2. van Asch CJ, Luitse MJ, Rinkel GJ, van der Tweel I, Algra A, Klijn CJ. Incidence, case fatality, and functional outcome of intracerebral haemorrhage over time, according to age, sex, and ethnic origin: a systematic review and meta-analysis. Lancet Neurol. 2010;9(2):167-76.

3. Rincon F, Mayer SA. Intracerebral hemorrhage: clinical overview and pathophysiologic concepts. Transl Stroke Res. 2012. doi:10.1007/s12975-012-0175-8.

4. Morgan T, Zuccarello M, Narayan R, Keyl P, Lane K, Hanley D. Preliminary findings of the minimally-invasive surgery plus rtPA for intracerebral hemorrhage evacuation (MISTIE) clinical trial. Acta Neurochir - Supplement. 2008;105:147-51.

5. Mendelow AD, Gregson BA, Mitchell PM, Murray GD, Rowan EN, Gholkar AR. Surgical trial in lobar intracerebral haemorrhage (STICH II) protocol. Trials. 2011;12:124.

6. Naff N, Williams MA, Keyl PM, Tuhrim S, Bullock MR, Mayer SA, et al. Low-dose recombinant tissue-type plasminogen activator enhances clot resolution in brain hemorrhage: the intraventricular hemorrhage thrombolysis trial. Stroke. 2011;42(11):3009-16.

7. Hanley DF. Intraventricular hemorrhage: severity factor and treatment target in spontaneous intracerebral hemorrhage. Stroke. 2009;40(4):1533-8.

8. Strahle J, Garton HJL, Maher CO, Muraszko KM, Keep RF, Xi G. Mechanisms of hydrocephalus after neonatal and adult intraventricular hemorrhage. Transl Stroke Res. 2012. doi:10.1007/ s12975-012-0182-9.

9. Bouz P. Neonatal intracerebral hemorrhage: mechanisms, managements and the outcomes. Transl Stroke Res. 2012 (in press).

10. Diringer MN, Skolnick BE, Mayer SA, Steiner T, Davis SM, Brun $\mathrm{NC}$, et al. Thromboembolic events with recombinant activated factor VII in spontaneous intracerebral hemorrhage: results from the Factor Seven for Acute Hemorrhagic Stroke (FAST) trial. Stroke. 2010;41(1):48-53.

11. Fisher M, Vasilevko V, Cribbs DH. Mixed cerebrovascular disease and the future of stroke prevention. Transl Stroke Res. 2012 (in press).

12. Keric N, Maier GS, Samadani U, Kallenberg K, Dehent P, Brueck $\mathrm{W}$, et al. Tissue plasminogen activator induced delayed edema in 
experimental porcine intracranial hemorrhage: reduction with plasminogen activator inhibitor-1 administration. Transl Stroke Res. 2012 (in press).

13. Woo D, Kaushal R, Chakraborty R, Woo J, Haverbusch M, Sekar $\mathrm{P}$, et al. Association of apolipoprotein E4 and haplotypes of the apolipoprotein E gene with lobar intracerebral hemorrhage. Stroke. 2005;36(9):1874-9.

14. Tzourio C, Arima H, Harrap S, Anderson C, Godin O, Woodward $\mathrm{M}$, et al. APOE genotype, ethnicity, and the risk of cerebral hemorrhage. Neurology. 2008;70(16):1322-8.

15. Lei B, Mace B, Bellows ST, Sullivan PM, Vitek MP, Lasowitz DT, et al. Interaction between sex and apolipoprotein E genetic background in a murine model of intracerebral hemorrhage. Transl Stroke Res. 2012 (in press).

16. Valant V, Keenan BT, Anderson CD, Shulman JM, Devan WJ, Ayres AM, et al. TOMM40 in cerebral amyloid angiopathy related intracerebral hemorrhage: comparative genetic analysis with Alzheimer's disease. Transl Stroke Res. 2012. doi:10.1007/ s12975-012-0161-1.

17. Xi G, Keep RF, Hoff JT. Mechanisms of brain injury after intracerebral haemorrhage. Lancet Neurol. 2006;5(1):53-63.

18. Johnston KC, Hall CE, Kissela BM, Bleck TP, Conaway MR, Investigators G. Glucose Regulation in Acute Stroke Patients (GRASP) trial: a randomized pilot trial. Stroke. 2009;40 (12):3804-9.

19. Feng W, Tauhid S, Goel S, Sidorov EV, Selim M. Hyperglycemia and outcome in intracerebral hemorrhage: from bedside to benchmore study is needed. Transl Stroke Res. 2012 (in press).

20. Bodmer D, Vaughan KA, Zacharia BE, Hickman ZL, Connolly ES. The molecular mechanisms that promote edema after intracerebral hemorrhage. Transl Stroke Res. 2012. doi:10.1007/s12975012-0162-0.

21. Liu D, Sharp FR. Excitatory and mitogenic signaling in cell death, blood-brain barrier breakdown and bbb repair after intracerebral hemorrhage. Transl Stroke Res. 2012. doi:10.1007/s12975-0120147-z.

22. Lok J, Zhao S, Leung W, Seo JH, Navaratna D, Wang X, et al. Neuregulin-1 effects on endothelial and blood-brain-barrier permeability after experimental injury. Transl Stroke Res. 2012. doi:10.1007/s12975-012-0157-x.

23. Yao Y, Tsirka SE. Chemokines and their receptors in intracerebral hemorrhage. Transl Stroke Res. 2012. doi:10.1007/s12975-0120155-z.

24. Hammond MD, Ai Y, Sansing LH. Gr1+ macrophages and dendritic cells dominate the inflammatory infiltrate 12 hours after experimental intracerebral hemorrhage. Transl Stroke Res. 2012. doi:10.1007/s12975-012-0174-9.

25. Lively S, Schlichter LC. Age-related comparisons of evolution of the inflammatory response after intracerebral hemorrhage in rats. Transl Stroke Res. 2012. doi:10.1007/s12975-012-0151-3.

26. Loftspring MC, Johnson HL, Johnson AJ, Clark JF. Depletion of GR-1-positive cells is associated with reduced neutrophil inflammation and astrocyte reactivity after experimental intracerebral hemorrhage. Transl Stroke Res. 2012. doi:10.1007/s12975-0120184-7.

27. Simard JM, Tosun C, Ivanova S, Kurland DB, Hong C, Radecki L, et al. Heparin reduces neuroinflammation and transsynaptic neuronal apoptosis in a model of subarachnoid hemorrhage. Transl Stroke Res. 2012. doi:10.1007/s12975-012-0166-9.

28. Sukumari-Ramesh S, Alleyne CH, Dhandapani KM. Astrogliosis: a target for intervention in intracerebral hemorrhage? Transl Stroke Res. 2012. doi:10.1007/s12975-012-0165-x.

29. Hasan DM, Amans M, Tihan T, Hess C, Guo Y, Cha S, et al. Ferumoxytol-enhanced MRI to image inflammation within human brain arteriovenous malformations: a pilot investigation. Transl Stroke Res. 2012 (in press).

30. Xie B, Miao P, Sun Y, Wang Y, Yang GY. Micro computed tomography for hemorrhage disruption of mouse brain vasculature. Transl Stroke Res. 2012. doi:10.1007/s12975-012-0164-y.

31. He Y, Karabiyikoglu M, Hua Y, Keep RF, Xi G. ischemic preconditioning attenuates brain edema after experimental intracerebral hemorrhage. Transl Stroke Res. 2012. doi:10.1007/s12975-0120171-z.

32. Morgenstern LB. Treatment of intracerebral hemorrhage - is the glass half full or half empty? Transl Stroke Res. 2012. doi:10.1007/ s12975-012-0179-4. 